\title{
Commentary
}

\section{From beast to bedside: a commentary}

Diabetes mellitus comprises a group of disorders, the common feature of which is hyperglycaemia. This may be due to near-absolute deficiency of insulin (Type 1 diabetes) or to relative deficiency of insulin (Type 2 diabetes).

The clinical signs and symptoms associated with Type 1 diabetes have long been known, but only recently has its autoimmune pathogenesis been recognised. Initial efforts to understand Type 1 diabetes focused on the biochemical and physiological hallmarks of near-absolute insulin deficiency and the early age at onset of the disease (hence the earlier nomenclature of juvenile-type or insulin-dependent diabetes). Subsequently, it was recognised from autopsies of children who died with acute onset diabetes that a mononuclear infiltration frequently surrounded the islets (insulitis). This key observation suggested the involvement of an inflammatory process in the pathogenesis. Bottazzo and Doniach then made the seminal discovery that islet-cell antibodies were present in children with the disease [1]. This observation, together with its association with other autoimmune endocrinopathies, led to the hypothesis that Type 1 diabetes was also an autoimmune disease. During the last 20 years, additional clinical observations have strengthened this hypothesis. The most compelling observation was the recurrence of diabetes following pancreas transplants in identical twins discordant for the disease [2].

There are still many impediments to the study of diabetes in human subjects. These include genetic heterogeneity, the long human lifespan, a "free-range" lifestyle, inaccessibility of tissues and organs, and, of course, ethical considerations. Animal models circumvent some of these problems, but obviously, extrapolation of findings from animals to man (and vice versa) is a treacherous undertaking. Nonetheless, the advantages inherent to animal experimentation have enabled

Received: 30 July 2004 / Accepted: 10 August 2004

Published online: 22 October 2004

(C) Springer-Verlag 2004 scientists to obtain valuable information relevant to disease pathogenesis.

In 1922, Banting, Best, Collip and associates published their epochal paper describing the cure of diabetes mellitus in a dog [3]. Both dogs and other animal species were then used to analyse biochemical, biological and physiological parameters of diabetes. It was not until 50 years later, however, that experimental animals became available to study the pathogenesis of Type 1 diabetes. One of the first models was experimental insulitis induced by the administration of insulin preparations in several animal species. This model system recapitulated the morphological finding of insulitis but rarely induced hyperglycaemia. Later, in mice, came descriptions of diabetes with a possible immune component following infection with encephalomyocarditis virus as well as following the administration of multiple low doses of streptozotocin, which induced both insulitis and hyperglycaemia [4].

Although spontaneous diabetes occurs in several animal species, the disease has been described in very few of these. In 1974 a rat at the BioBreeding Laboratories in Canada was shown to become spontaneously diabetic in the context of insulitis and ketoacidosis. A few years later a mouse that also developed spontaneous diabetes and insulitis was discovered in Japan. These two models, the BioBreeding (BB) rat and the non-obese diabetic (NOD) mouse, have become the most important models of Type 1 diabetes [5]. Research is more advanced in the NOD mouse, as transgenic and knock-out technologies as well as a wide variety of species-specific reagents are available in this animal. During the past two decades, thousands of publications have appeared providing new immunological hypotheses and novel therapeutic intervention strategies. Now there is controversy, and the following proposal has been put forward: "Animal models have little to teach us about Type 1 diabetes."

The argument in support of this proposal, espoused by Roep and Atkinson [6], and the contrary position, 
by Leiter and von Herrath [7], are actually not too dissimilar. As we embark on studies from bench to bedside, e.g. from mouse to human, we need to take great care not to over-interpret the lesson that the rodent models provide. In both the BB rat, and later, the NOD mouse, bone marrow transplantation adoptively transferred the disease to a naive recipient. This experiment has unfortunately been recapitulated in a clinical setting [8].

Immunosuppressive agents such as cyclosporin were used successfully to prevent diabetes in the BB rat. This observation led to a clinical trial in new-onset Type 1 diabetes. Although this immunosuppressive agent preserved beta cell function in humans, nephrotoxicity precluded prolonged use or additional trials. Other strategies, including the administration of insulin or nicotinamide, were very effective in NOD mice, but clinical trials revealed no benefit in humans [5].

Thus, some agents may be helpful in animal models but the response can be species specific. The lymphopenia of the diabetes-prone BB rat and the many immune and functional abnormalities found in the NOD mouse make these animals far less similar to most cases of human Type 1 diabetes than we might wish.

When the great mammalian radiation occurred some 80 million years ago, humans and rodents diverged and random forces influenced their evolution. The divergence of rats and mice 10 to 15 million years ago was also seen to parallel the separation of their immunological characteristics [9]. An important point to make is that the immune systems of these species all evolved to defend that individual from its specific environmental danger. However, certain primitive responses have persisted throughout evolution. This is supported by the innate immune response against microbial infection, which is relatively non-specific. This innate immune response affects the adaptive immune response, which is the key factor responsible for autoimmunity. Adaptive immunity features the development of immunological memory, which is selective and individual. Additional factors that influence the immune response include the selection of mice by physical characteristics, inbreeding, and environment. Various environmental factors influence gene expression without changing gene sequences (epigenetic inheritance). For example, folic acid fed to a pregnant agouti mouse affects the colour pattern of hair in the offspring and possibly affects immunological patterns [10]. Because spontaneous insulin-deficient diabetes is present in so many species, the question remains as to whether there is an advantage to having diabetes, or is it simply collateral damage? Is the beta cell the innocent target of a hyper-responsive gene complex that offers protection against environmental perturbants [11]?

Animals only provide us with lessons; they are not exact models of human Type 1 diabetes [12]. Reagents in rodents may recognise different epitopes in humans, and paradigms in rodents may be less clearly defined in humans. However, we should not throw the baby out with the bathwater just because some findings in NOD mice do not mirror those in humans. We should proceed with caution, but should not discontinue the use of this valuable animal model. That experiments should be examined in more than one model is crucial, as the reviewers suggest. We should also be sceptical whenever a new rodent treatment modality is discovered; it will need confirmation.

Obviously, we only really care about diabetes in people, but the intensity of this caring pushes observations that are inchoate in all species into clinical trials. In reality, the pressure to do so comes not just from caring; it is also the result of many other factors:

Economic. Successful therapies today inevitably have great financial implications for discoverers, laboratories, universities, corporations and funding institutions.

Political. Legislators and administrations need to justify the use of tax money for basic research. Is it easier to spend on rodent studies where lessons have still not panned out, or on a hopeful human trial?

Organisations. Promises are made for a cure which may be difficult to achieve on a certain timetable.

Media. It is not difficult to imagine that a constant diet of major breakthrough discoveries and plans for human cures are desired to sell newspapers and to increase the impact of TV advertisements.

Marketing. Institutions are addicted to philanthropy that is attracted to the good news of promising, if not definitive breakthroughs.

Patients and family members who suffer from the ravages of diabetes. They provide the most pressure, pleading with the scientist to find a cure. We now live in an era where great scientific discoveries and technologies make a cure a real possibility. However, the issue of equipoise should always be carefully considered. Will the cure cause more harm than the disease?

The NOD mouse and BB rat currently provide much scientific insight into the autoimmune pathogenesis of human diabetes, but this knowledge is currently imprecise. Animals are important for testing therapeutic strategies, and can be used to determine dosage, immune responses, and potentially, unique responses, but a single observation should not be sufficient to justify a clinical trial. The challenge should not be how quickly we can get to the clinical trial (bedside) but rather how an observation can be explained and used to develop experiments to describe the mechanisms. Use of other animal models may also be useful. 
As the Italian proverb goes: Chi va piano va sano e va lontano (He who goes slowly goes safely and goes far).

\section{A. A. Rossini \\ Diabetes Division, Department of Medicine, The University of Massachusetts Medical School, Worcester, USA}

Acknowledgements. A. A. Rossini is supported in part by grants DK25306, DK56946, AI46629 and DK53006, by an institutional Diabetes Endocrinology Research Center (DERC) grant (DK32520) from the National Institutes of Health, by a Mentor-Based Postdoctoral Fellowship from the American Diabetes Association, and by a fellowship grant from the Iacocca Foundation.

\section{References}

1. Bottazzo GF, Florin-Christensen AF, Donaich D (1974) Islet cell antibodies in diabetes mellitus with autoimmune polyendocrine deficiencies. Lancet 2:1279-1282

2. Sutherland DE, Sibley R, Xu XZ et al. (1984) Twin-to-twin pancreas transplantation: reversal and reenactment of the pathogenesis of type I diabetes. Trans Assoc Am Physicians 97:80-87

3. Banting FG, Best DH, Collip JB, Campbell WR, Fletcher AA (1922) Pancreatic extracts in the treatment of diabetes mellitus. Preliminary report. CMAJ 22:141-146

4. Rossini AA, Mordes JP (1992) Insulin dependent diabetes mellitus, experimental models. In: Roitt IM (ed) Encyclopedia of immunology. Academic Press, San Diego, pp 872-877

5. Mordes JP, Serreze DV, Greiner DL, Rossini AA (2004) Animal models of autoimmune diabetes mellitus. In:
LeRoith D, Taylor SI, Olefsky JM (eds) Diabetes mellitus. A fundamental and clinical text, 3rd edn. Lippincott Williams \& Wilkins, Philadelphia, pp 591-610

6. Roep BO, Atkinson M (2004) Animal models have little to teach us about Type 1 diabetes: 1 . In support of this proposal. Diabetologia DOI 10.1007/s00125-004-1517-1

7. Leiter EH, Herrath M von (2004) Animal models have little to teach us about Type 1 diabetes: 2. In opposition to this proposal. Diabetologia DOI 10.1007/s00125-0041518-0

8. Sorli CH, Greiner DL, Mordes JP, Rossini AA (1998) Stem cell transplantation for treatment of autoimmune diseases. Graft 1:71-81

9. Makalowski W, Boguski MS (1998) Evolutionary parameters of the transcribed mammalian genome: an analysis of 2,820 orthologous rodent and human sequences. Proc Natl Acad Sci USA 95:9407-9412

10. Waterland RA, Jirtle RL (2003) Transposable elements: targets for early nutritional effects on epigenetic gene regulation. Mol Cell Biol 23:5293-5300

11. Rossini AA, Mordes JP, Handler ES (1988) Speculations on etiology of diabetes mellitus: tumbler hypothesis. Diabetes 37:257-261

12. Renold AE, Porte D Jr, Shafrir E (1988) Definitions for diabetes types: use and abuse of the concept 'animal models of diabetes mellitus'. In: Shafrir E, Renold AE (eds) Frontiers in diabetes research. Lessons from animal diabetes II. John Libbey \& Co, London, pp 3-7

A. A. Rossini $(\bullet)$

Diabetes Division, Department of Medicine,

The University of Massachusetts Medical School, 373 Plantation Street, Biotech 2, Suite 218,

Worcester, MA 01605, USA

E-mail: aldo.rossini@umassmed.edu

Tel.: +1-508-8563800, Fax: +1-508-8564093 